\title{
Impactos da inflação sobre a desigualdade de renda ${ }^{\dagger}$
}

\author{
Ricardo Sabbadini* \\ Mauro Rodrigues ${ }^{* *}$
}

RESUMO - O objetivo deste artigo é avaliar o impacto de uma mudança da taxa de inflação sobre a desigualdade de renda, medida pelo índice de Gini. Para isso, usa-se um painel de aproximadamente 80 países, com dados anuais entre 1987 e 2006. Estimam-se modelos econométricos em que a desigualdade de renda é explicada pela inflação, mantendo sempre um controle baseado na existência de efeitos fixos para países e anos. Quando modelos não-lineares são usados, encontra-se uma robusta relação positiva entre as variáveis, indicando que a inflação tem um impacto positivo sobre o índice de Gini. A magnitude do efeito estimado, porém, é bastante inferior à obtida por estudos anteriores. Os resultados apontam que um aumento na taxa de inflação de zero para 10 pontos percentuais ao ano elevaria o índice de Gini (que está numa escala de zero a 100) em, no máximo, 0,05 pontos percentuais. Essa discrepância em relação à literatura parece decorrer do uso de estimadores de efeitos fixos, pois os trabalhos anteriores baseavam-se em dados em cross section.

Palavras-chave: Inflação. Desigualdade de renda. Dados em painel.

\section{INTRODUÇÃO E OBJETIVOS}

Nos debates econômicos sobre os malefícios de elevadas taxas de inflação, é recorrente o argumento de que o aumento do nível de preços afeta mais fortemente os pobres. Este efeito desigual seria originário do acesso diferenciado a ativos financeiros indexados entre pobres e ricos. Desta maneira, os mais abastados podem se proteger conforme a inflação se eleva, diferentemente da parte mais pobre da sociedade. Assim, a inflação levaria ao aumento da desigualdade de renda, sendo este mais um bom motivo para sua manutenção em baixos níveis. Recentemente, este argumento foi inclusive formalizado em modelos dinâmicos de equilíbrio geral ${ }^{1}$.

Com base em dados de cross sections de países, trabalhos empíricos anteriores encontraram uma forte relação entre inflação e desigualdade de renda. Romer e Romer (1998), por exemplo, sugerem que um aumento da inflação em 10 pontos percentuais poderia elevar o ín-

\footnotetext{
† Este texto é uma versão expandida de artigo publicado no Boletim de Informações da Fipe (edição no. 356, maio de 2010), e baseia-se nos resultados da dissertação de mestrado de Ricardo Sabbadini, defendida em março de 2010 no Departamento de Economia da FEA/USP (SABBADINI, 2010).

*Mestre em Teoria Econômica pela FEA/USP, analista do Banco Central do Brasil. Endereço eletrônico: ricardo. sabbadini@usp.br.

${ }^{* *}$ Doutor em economia pela Universidade da Califórnia em Los Angeles (UCLA). É professor do Departamento de Economia da FEA/USP. Endereço eletrônico: mrodrigues@usp.br.

1 Veja, por exemplo, Erosa e Ventura (2002), Cysne, Maldonado e Monteiro (2005) e Albanesi (2007).
} 
dice de Gini em até 3,4 pontos, quando esta variável encontra-se em uma escala de 0 a $100^{2}$. Tal abordagem, todavia, sofre potencialmente de sérios problemas de variável omitida. Por exemplo, países com instituições ruins tendem tanto a serem mais desiguais (pois o acesso ao poder público é restrito a uma pequena elite), como apresentarem taxas de inflação mais altas (já que a baixa capacidade da população de cobrar seus governantes favorece a má gestão macroeconômica). Nesse sentido, a correlação positiva observada entre inflação e desigualdade seria causada por um terceiro fator (instituições de baixa qualidade), não havendo necessariamente uma relação causal direta entre as duas variáveis.

No presente artigo, buscamos mitigar estes problemas de omissão de variáveis ao utilizar um painel de países. Mais precisamente, nossa análise permite controlar por características não observáveis de cada país, mas que mudam pouco no tempo. Nossos resultados apontam para um efeito positivo da inflação sobre a desigualdade de renda, mas apenas quando modelos não-lineares são considerados. Entretanto, a magnitude deste efeito é muito menor do que a encontrada por estudos empíricos anteriores, os quais utilizavam dados em cross section. Em particular, um aumento na taxa de inflação de zero para 10 pontos percentuais ao ano elevaria o índice de Gini em, no máximo, 0,05 pontos percentuais.

\section{DADOS}

A fim de avaliar o impacto da inflação sobre a desigualdade de renda, é necessário analisar dados de países, pois apenas para estes existem medidas tanto do aumento do nível de preços como da distribuição de renda. Nesse sentido, utilizamos um painel de 81 países, com dados anuais de 1987 e 2006.

Para a desigualdade de renda, recorreu-se ao seu indicador mais difundido, o índice de Gini. Sua origem é a World Income Inequality Database (WIID) versão 2c do World Institute for Development Economics Research (WIDER), United Nations University, uma base de dados secundária que compila e classifica informações sobre distribuição de renda de vários países. Quanto maior este indicador, limitado entre zero e 100, mais desigual a economia ${ }^{3}$.

A taxa de inflação, variável explicativa de interesse, é medida pela variação anual do índice de preços ao consumidor (IPC), sendo os dados obtidos na base International Financial Statistics (IFS) do Fundo Monetário Internacional (FMI). Da base World Development Indicators 2007, compilada pelo Banco Mundial, foram retiradas as demais variáveis explicativas de con-

2 Veja também Easterly e Fischer (2001) e Bulír (2001).

3 Sobre indicadores de desigualdade de renda, inclusive o índice de Gini, é importante ressaltar que existem diversas dificuldades metodológicas em seu cálculo e diferentes métodos de obtê-los. Para obter os detalhes do tratamento desses dados em nosso caso, veja Sabbadini (2010). 
trole utilizadas na estimação dos modelos econométricos, a saber: taxa de crescimento do PIB, PIB per capita e seu quadrado, abertura ao comércio internacional, crescimento populacional e crédito doméstico ao setor privado como porcentagem do PIB. A escolha destas variáveis foi baseada na literatura sobre determinantes da desigualdade de renda.

As estatísticas descritivas detalhadas do índice de Gini e da taxa de inflação estão na Tabela 1. O índice de Gini está em uma escala entre zero e 100, com média 37,42 e mediana 34,5. As menores estatísticas, 19,7, ficam por conta da Finlândia em 1987 e 1992, e a maior se refere ao Equador em 2004. O índice de inflação utilizado, baseado no IPC, tem média de $48,1 \%$ ao ano, mas mediana de apenas 4,4\%. Essa discrepância ocorre em virtude de algumas observações extremamente elevadas. Enquanto no percentil 95 a inflação está em 63,9\% ao ano, no percentil 99 ela é 1190,2\%. A inflação não é apresentada em porcentagem a fim de facilitar a interpretação dos coeficientes obtidos na seção de resultados.

TABELA 1 - ESTATÍSTICAS DESCRITIVAS PARA GINI E INFLAÇÃO

\begin{tabular}{|c|c|c|}
\hline & Gini & IPC \\
\hline Média & 37,42 & 0,481 \\
\hline Desvio-Padrão & 10,27 & 3,365 \\
\hline Mínimo & 19,70 & $-0,114$ \\
\hline Máximo & 62,83 & 52,442 \\
\hline $10^{\circ}$ percentil & 25,90 & 0,012 \\
\hline $1^{\circ}$ Quartil & 26,90 & 0,023 \\
\hline Mediana & 34,50 & 0,044 \\
\hline $3^{\circ}$ Quartil & 45,80 & 0,100 \\
\hline $90^{\circ}$ percentil & 53,70 & 0,283 \\
\hline $95^{\circ}$ percentil & 56,43 & 0,639 \\
\hline $99^{\circ}$ percentil & 60,05 & 11,902 \\
\hline
\end{tabular}

\section{METODOLOGIA}

Em todas as estimativas apresentadas neste artigo, os estimadores levam em conta os efeitos fixos dos países e incluem dummies de tempo (para controlar por choques agregados comuns aos países). Assim, em virtude do uso deste tipo de estimador, as estimativas são baseadas na variação dos dados dentro de um mesmo país (within-country) e não entre eles.

Além disso, com o uso de estimadores de efeitos fixos, pode-se reduzir o viés causado pela existência de variáveis relevantes omitidas, que potencialmente ocorre nos estudos anteriores baseados em dados de cross section. Caso estas variáveis omitidas variem pouco no tempo, podemos aproximá-las por um termo de heterogeneidade não-observada para cada país (isto é, um efeito fixo), mitigando assim esse problema que causa endogeneidade e impede a obtenção 
de estimadores consistentes.

Um exemplo ajuda a esclarecer este ponto. Países com instituições de baixa qualidade - pois herdaram de seus colonizadores instituições que não coíbem governantes de se apropriar da riqueza produzida - tendem a possuir maiores níveis de desigualdade. Ademais, estas instituições também podem levar a governos maiores e mais engajados em rent seeking, que se financiam excessivamente com emissão de moeda. Dessa forma, a inflação estaria correlacionada com estas instituições que também afetam a desigualdade de renda, enviesando as estimativas baseadas em dados de cross section em virtude da existência de variáveis omitidas. Como instituições tendem a variar pouco no tempo, a utilização de uma estrutura de painel (e o consequente controle por efeitos fixos) permite corrigir pelo menos em parte este problema de endogeneidade, levando a estimativas mais precisas quanto ao efeito causal da inflação sobre a desigualdade de renda.

Além disso, inspirado em resultados de modelos teóricos, utiliza-se uma forma funcional flexível (denotada pela função $\mathbf{f}()$ ), que permite um efeito não linear da inflação (representada aqui por $\mathbf{I P C}_{\mathrm{it}}$ ) sobre a desigualdade de renda $\left(\mathbf{G i n i}_{\mathrm{it}}\right)$, como apresentado na equação abaixo, em que $\mathbf{a}_{\mathbf{i}}$ é o efeito fixo do país i, $\mathbf{b}_{\mathbf{t}}$ é o valor da dummy para o ano t e $\boldsymbol{\alpha}$ é o vetor de parâmetros de interesse.

$$
\text { Gini }_{i t}=a_{i}+b_{t}+\alpha f\left(\text { IPC }_{i t}\right)+\boldsymbol{\beta C o n t r o l e s}_{i t}+v_{i t}
$$

Neste artigo, consideramos não apenas um formato linear para a função, mas também quadrático, possibilitando que o efeito do IPC sobre a desigualdade de renda mude de intensidade e até mesmo de sinal de acordo com o nível da inflação. Consideramos, ainda, intervalos para a taxa de inflação, em que a relação é linear dentro de cada intervalo, mas com inclinações diferentes para intervalos distintos. Isso é obtido usando como regressores o IPC e interações suas com dummies que definem os intervalos onde a inclinação muda. Os nós que definem tais intervalos nesse estudo são 40\%, 100\% e 1000\%.

A estimação se dá pelo método de efeitos fixos, sendo a inferência baseada em errospadrão robustos (que permitem heteroscedasticidade e autocorrelação nos erros dentro de cada país). Nesse caso, a hipótese identificadora é que a inflação ou sua transformação são variáveis exógenas, isto é, não correlacionadas com o termo de erro $\mathbf{v}_{\mathrm{it}}$. Porém, elas podem ser livremente correlacionadas com os termos de efeito fixo ${ }^{4}$.

4 Em Sabbadini (2010) outros métodos de estimação também são utilizados. Alguns deles, inclusive, permitem que a inflação seja endógena. Os resultados, porém, são bastante semelhantes aos expostos neste artigo. 


\section{RESULTADOS}

Para fins de comparação, é importante apresentar o principal resultado anterior desta literatura. Romer e Romer (1998) encontram, em um modelo linear com dados em cross section, que uma elevação da inflação em um ponto percentual aumenta o Gini em 0,34 pontos. Dessa forma, um incremento de 10 pontos percentuais da inflação elevaria o Gini em 3,4 pontos percentuais.

Vejamos agora os resultados do presente estudo, expostos na Tabela 2. No modelo linear (coluna 1), parece não haver uma relação entre o índice de Gini e a inflação, pois não é possível distinguir os coeficientes estimados de zero. Além disso, em oposição à teoria, o coeficiente estimado é negativo, porém a magnitude dos efeitos é bastante reduzida: um aumento da inflação em 100 pontos percentuais altera o índice de Gini, que está em uma escala entre zero e 100 , em aproximadamente 0,0781 .

TABELA 2 - RESULTADOS DOS MODELOS ECONOMÉTRICOS

\begin{tabular}{|c|c|c|c|c|}
\hline & 1 & 2 & 3 & 4 \\
\hline IPC & $\begin{array}{l}-0,0781 \\
(0,0681)\end{array}$ & & $\begin{array}{r}0,302 * * * \\
(0,104)\end{array}$ & $\begin{array}{r}0,419 * * * \\
(0,135)\end{array}$ \\
\hline Imposto Inflacionário & & $\begin{array}{l}-1,131 \\
(2,098)\end{array}$ & & \\
\hline $\mathrm{IPC}^{2}$ & & & $\begin{array}{r}-0,00857 * * * \\
(0,00219)\end{array}$ & \\
\hline $\mathrm{D}[40<\mathrm{IPC}<100] *(\mathrm{IPC}-40)$ & & & & $\begin{array}{l}2,683^{*} \\
(1,525)\end{array}$ \\
\hline $\mathrm{D}[100<\mathrm{IPC}<1000]^{*}(\mathrm{IPC}-100)$ & & & & $\begin{array}{r}-0,509^{*} \\
(0,301)\end{array}$ \\
\hline $\mathrm{D}[\mathrm{IPC}>1000]^{*}(\mathrm{IPC}-1000)$ & & & & $\begin{array}{r}-0,660 * * * \\
(0,165) \\
\end{array}$ \\
\hline 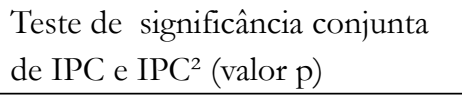 & - & - & 0,000 & - \\
\hline $\mathrm{IPC}+\mathrm{D}[40<\mathrm{IPC}<100]^{*}(\mathrm{IPC}-40)$ & & & $3,102^{* *}$ & \\
\hline $\mathrm{IPC}+\mathrm{D}[\mathrm{IPC}>100] *(\mathrm{IPC}-100)$ & & & 0,78 & \\
\hline $\mathrm{IPC}+\mathrm{D}[\mathrm{IPC}>1000]^{*}(\mathrm{IPC}-1000)$ & & & $-0,241 * * *$ & \\
\hline
\end{tabular}

NOTA: Desvios-padrão robustos a heterocedasticidade e autocorrelação em parênteses. Todos os modelos incluem as variáveis de controle apresentadas no texto. $\mathrm{D}[40<\mathrm{IPC}<100]$ representa uma variável dummy igual a 1 se a inflação encontra-se entre 40 e 100\%, e zero caso contrário (analogamente para D $[100<\mathrm{IPC}<1000]$ e $\mathrm{D}[\mathrm{IPC}>1000]) .{ }^{* * *} \mathrm{p}<0.01,{ }^{* *} \mathrm{p}<0.05,{ }^{*} \mathrm{p}<0.1$.

A coluna 2 apresenta os resultados da estimação do modelo em que o Gini depende de forma não-linear da inflação, mas linearmente de uma medida do imposto inflacionário, dada por $\left(\mathbf{I P C}_{\mathrm{it}}\right) /\left(\mathbf{1}+\mathbf{I P C}_{\mathrm{i}}\right)$, como usado por Easterly e Fischer (2001). Nesse modelo, todos os resultados qualitativos da coluna 1 são mantidos. 
Por outro lado, quando estimamos modelos não-lineares, obtemos efeitos positivos e significantes da inflação sobre o índice de Gini. No modelo quadrático (coluna 3), os coeficientes do IPC e de seu quadrado são individual e conjuntamente significantes a 1\%. Enquanto o termo linear é positivo, o quadrático sempre é menor que zero. Em outras palavras, a relação estimada entre Gini e IPC tem a forma de um "U” invertido. Assim, até certo nível de inflação, esta possui um efeito positivo no Gini, piorando a distribuição de renda. Para valores maiores de inflação, contudo, o Gini é negativamente afetado pela inflação. Porém, o valor do IPC a partir do qual a inflação tem um efeito negativo sobre o Gini (isto é, passa a melhorar a distribuição de renda) é muito elevado, $1761 \%$ ao ano. Como o $99^{\circ}$ percentil do IPC possui o valor de $1190 \%$, a inflação tem um efeito negativo sobre o Gini para menos de 1\% da amostra. Assim, para a maioria dos países existe uma relação positiva entre inflação e desigualdade de renda.

Como a relação estimada tem a forma de "U" invertido, a magnitude do efeito marginal é decrescente no IPC, conforme descrito no Gráfico 1. Em particular, a alteração da inflação de zero para 10\% ao ano aumentaria o índice de Gini em 0,03 pontos percentuais. A importância econômica deste efeito é substancialmente inferior ao que estudos prévios sugerem, mas sem dúvida é significante.

\section{GRÁFICO 1 - IMPACTO DA INFLAÇÃO SOBRE O GINI SEGUNDO O MODELO QUADRÁTICO}

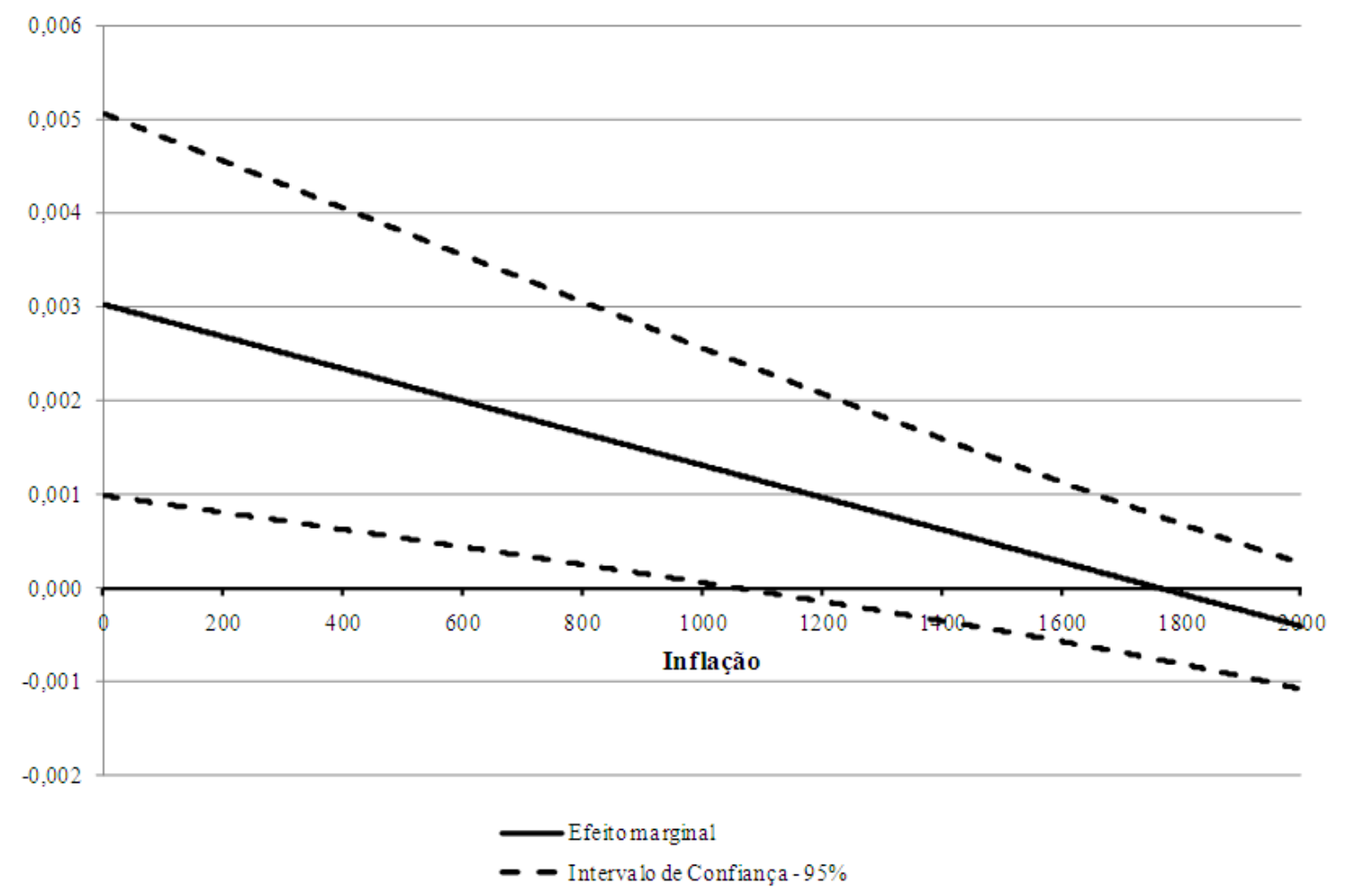

Outro exercício quantitativo interessante é obter a diferença entre o Gini previsto de 
um país com inflação igual a 1761\% (valor correspondente ao ápice do "U” invertido estimado) e com inflação nula. Este seria o maior efeito possível sobre o índice de Gini de uma redução para zero na inflação. Nossa estimativa é que este efeito seria de apenas 2,66 pontos percentuais no Gini.

Portanto, o modelo quadrático mostra que existe um impacto positivo, porém pequeno, da inflação sobre o Gini. Outro ponto importante é que este efeito não é positivo para inflações acima de $1761 \%$ ao ano. Como esta conclusão se baseia em um número reduzido de observações (menos de 1\% da amostra tem inflação superior a este número) e o intervalo de confiança a 95\% inclui o zero, como pode ser visto no Gráfico 1, não é apropriado afirmar que para inflações elevadas este efeito seja negativo, pois não se pode distingui-lo do zero. É possível que em situações com inflação tão alta, os efeitos distributivos máximos já tenham ocorrido. Pode ser que nesses casos extremos a moeda já tenha perdido suas funções, sendo substituída por outro meio de troca (o dólar, por exemplo) ou que nesse ponto a economia já esteja completamente indexada. Neste último caso, com a maioria dos ativos atrelados à inflação, é possível que a diferença no acesso a ativos indexados entre pobres e ricos diminua, minimizando os impactos distributivos do aumento generalizado de preços.

Agora consideramos uma forma alternativa de não-linearidade, a qual permite que o efeito marginal varie de acordo com quatro intervalos distintos: taxa de inflação menor que $40 \%$, entre 40 e $100 \%$, entre 100 e 1000\%, e superior a 1000\%. Para tanto, interagimos o IPC com dummies que representam tais intervalos. Ou seja, o efeito marginal é o mesmo dentro de cada intervalo, mas pode diferir entre intervalos. Os resultados encontram-se na coluna 4 da Tabela 1. Para o grupo base (com IPC abaixo de 40\%), que inclui mais de $90 \%$ da amostra, encontra-se uma relação positiva e significante a 1\%. A alteração do IPC de zero para $40 \%$ elevaria o Gini em apenas 0,17 pontos percentuais.

Para o segundo intervalo, de 40 a 100\%, o efeito da inflação sobre a distribuição de renda é significante a $5 \%$ e possui magnitude de 3,1, bem acima daquele do primeiro intervalo $(0,419)$. Desse modo, a mudança da inflação de $40 \%$ para $100 \%$ piora o Gini em 1,86 pontos percentuais. Portanto, este modelo indica que um aumento da inflação de zero para 100\% elevaria o Gini em 2,03 pontos. Entre 100\% e 1000\%, as estimativas do efeito marginal não podem ser estatisticamente distinguidas de zero. Para o último intervalo, onde resta pouco mais de 1\% da amostra, encontra-se uma relação negativa. Entretanto, deve-se lembrar que essa estimativa é baseada em pouquíssimas observações, como comentado na interpretação dos modelos quadráticos. Por fim, a principal mensagem desse modelo com dummies é referente aos valores mais baixos da inflação. Quando se controla a presença dos outliers, fica evidente o impacto positivo 
da inflação no Gini. Ademais, esse modelo indica que o efeito pode ser mais nocivo com a inflação alta (entre 40\% e 100\%) do que baixa.

Embora o efeito captado aqui nos modelos quadráticos e com dummies esteja abaixo do obtido por trabalhos anteriores, argumentamos que ele deve estar mais próximo do efeito causal verdadeiro. Além dos motivos apresentados nas seções sobre dados e metodologia, uma breve análise de alguns episódios de inflações altas reforça essa ideia. Segundo a nossa base de dados, no Brasil entre 1990 e 2005, o Gini caiu apenas 3,94 pontos percentuais mesmo após o fim de uma inflação superior a $2000 \%$ ao ano, e sem considerar várias outras alterações na economia que podem ter ajudado nesta queda, como abertura ao comércio internacional e aumento nos benefícios sociais trazidos pela Constituição de 1988. Entre 1997 e 1998, a inflação na Bulgária diminuiu de 1058\% para 18\%, mas o Gini caiu apenas 2,1 pontos. Na Rússia, em 1993 a inflação superou os $800 \%$ ao ano e o índice de Gini era de 46,1 pontos. Treze anos depois, com uma inflação abaixo de 10\%, o Gini era igual a 45,1. Se as estimativas de Romer e Romer (1998) fossem corretas, o Gini das economias citadas acima teria diminuído muito mais.

\section{CONCLUSÕES}

Utilizando dados em painel e formas funcionais não-lineares, encontramos evidências de um efeito positivo da inflação sobre o índice de Gini para a maior parte de nossa amostra. A magnitude desse efeito, todavia, é muito menor do que a encontrada por trabalhos anteriores, que se baseavam em dados de cross section.

Vale notar que os resultados aqui apresentados, em sintonia com a experiência de países como Brasil e Rússia, mostram que inflações altas têm impactos negativos e não desprezíveis sobre a distribuição de renda. Contudo, eles indicam que não é possível alterar completamente um padrão histórico de desigualdade de renda apenas debelando ou criando um episódio de inflação elevada ou hiperinflação.

\section{REFERÊNCIAS}

ALBANESI, Stefania. Inflation and inequality. Journal of Monetary Economics, v. 54, n. 4, p. 1088-1114, 2007.

BULÍR, Ales. Income inequality: does inflation matters? IMF Staff Papers, v. 48, n. 1, p.139159, 2001.

CYSNE, Rubens Penha; MALDONADO, Wilfredo; MONTEIRO, Paulo Klinger. Inflation and income inequality: a shopping-time approach. Journal of Development Economics, v. 78, p. 516-528, 2005. 
EASTERLY, William; FISCHER, Stanley. Inflation and the poor. Journal of Money, Credit and Banking, v. 33, n. 2, p. 160-178, 2001.

EROSA, Andrés; VENTURA, Gustavo. On inflation as a regressive consumption tax. Journal of Monetary Economics, v. 49, n. 4, p. 761-795, 2002.

ROMER, Christina; ROMER, David. Monetary policy and the well-being of the poor. NBER Working Paper Series, n. 6793, 1998.

SABBADINI, Ricardo. Dois ensaios empíricos em macroeconomia e desigualdade de renda. Dissertação (Mestrado em Economia) - Instituto de Pesquisas Econômicas, Universidade de São Paulo. São Paulo, 2010. 
